\title{
Hybrid Design and RF Planning for 4G networks using Cell Prioritization Scheme
}

\author{
Dr. S.A. Sivakumar \\ Assistant professor-ECE, Info Institute of Engineering \\ drsasivakumar@gmail.com
}

\begin{abstract}
This article includes hands-on recreation practice on arranging of RF connect with the assistance of Atoll arranging programming device. The primary goal of this task is to structure and plan a RF coordinate with obstruction free correspondence, Optimum inclusion, no forgot about zone in the arranged inclusion guide and extension and reuse of site recurrence \&network structure. Using the accessible restricted data transfer capacity vitally in order to take into account millions out of an immense zone with great quality, inclusion, and without obstruction utilizing ATOLL arranging device. Insightful re-utilization of site area later on organize structure will set aside cash for the administrator. Handover component is critical in cell arrange in light of the cell engineering utilized to expand range usage. One approach to improve the phone organize execution is to utilize productive handover prioritization plans, which have a typical trademark lessening the call dropping likelihood to the detriment of expanded call blocking likelihood. Effective prioritization conspire obliges various new calls while ensures the nature of administration (QOS) of Hand over call. This thought depends on the neighboring cells have a covering (the territory served by more than one cell) inclusion zone. Moreover cell cover and burden adjusting plan is proposed to improve the GSM cell limit utilizing a Software advancement pack (SDK). Limit improvement is accomplished by adjusting the heap in neighboring cell prioritization plans when client is exchanging between the cells.
\end{abstract}

Keywords: RF network, ATOLL tool, Handover, SDK, QoS

\section{Introduction}

System RF arranging is the establishment of versatile correspondence organize. Accomplishing most extreme limit while keeping up a worthy evaluation of administration and great discourse quality is the principle issue for the system arranging. Arranging a youthful system with a predetermined number of supporters isn't the issue. The trouble is to design a system that follows future development. Shrewd re-utilization of site area later on arrange structure will set aside cash for the system. At present the manual method of monitoring the planned coverage map to avoid interference and handover problems is adopted. But it is not that much efficient [1].

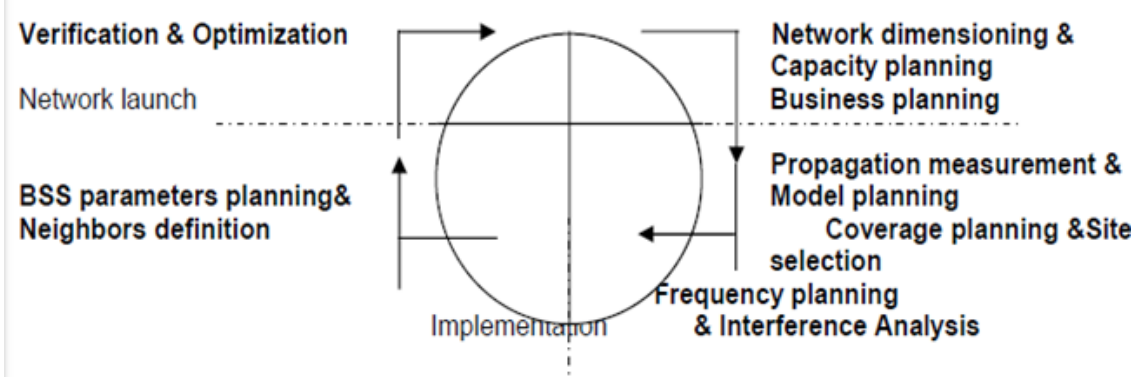

Fig 1: Basic structure of Radio network planning

The obligations of MSC are to investigate call goals, course calls, taking care of flagging, control handover, pack and tomb voice. These obligations are done physically through the data given from the digitized inclusion map. Yet at the same time impedance and hands over issues are not cleared because of manual figurings and likelihood. GSM radio system arranging is to give a financially savvy answer for the radio system. Radio arranging and looking over activities are interlinked in a cordial iterative procedure that ought to at last lead to 
the individual site plan, in which the effectiveness can't be acquired through the manual works. Along these lines, the mechanization of versatile exchanging focus (MSC) is important [2].

System structure, as it needs to work in close coordination with the arranging procedures of the center and particularly the transmission organize. In any case, for simplicity of clarification, an improved procedure only for radio system arranging is appeared in above Figure. The arranging and the enhancement procedure can likewise be mechanized with insightful instruments and system components. They are as per the following:
a) Capacity Planning
b) Coverage Planning
c) Parameter Planning
d) Frequency Planning

Traffic scope organization is a difficult assignment in different information sources numerous yield (MIMO) and symmetrical recurrence division (OFDM) based long haul advancement (LTE) cell systems, came about because of rising various interactive media traffic necessity, along with exceptionally open and adaptable air interface structure in LTE. In this paper, another strategy for dynamic constant scope quantification is proposed for LTE radio system dimensioning, in view of bound together traffic process component, new reproduction procedure for air interface, and savvy self-assessment and enhancement. By relating programming structure and usage, it gives useful asset to LTE organize organizers to get proficient, exact and proficient scope organization result absent a lot of manual exertion [3].

The limit of a given system is estimated as far as the endorsers or the traffic load that it can deal with. The previous requires information on supporter calling propensities (normal traffic per endorser) while the last is increasingly broad. The goal of inclusion arranging stage in inclusion restricted system regions is to locate a base measure of cell destinations with ideal areas for delivering the necessary inclusion for the objective region. Inclusion arranging is ordinarily performed with forecast modules on advanced guide database. Inclusion arranging and site determination are performed on corresponding with the site securing in intelligent mode. Both system arranging group and site securing should group have all around characterized obligations and intends to impart [4].

The Broadcast channels send data on the phone properties, for example, synchronization, recurrence revision, utilized frequencies and force levels, neigh exhausting cells [5]. There are three distinctive communicate control channels $(\mathrm{BCCH})$. The regular control channels are utilized while setting up a flagging association between the MS and BTS. The paging channel (PCH) is utilized when BTS needs to contact the MS. The MS demands a flagging channel on an irregular access channel (RACH). The flagging channel is dispensed to the MS by utilizing Access award channel (AGCH).

\section{Existing techniques}

System RF arranging is the establishment of a portable correspondence organize. Arranging of RF organize is finished with the assistance of Atoll arranging programming instrument. The primary point of radio system arranging is to give a practical answer for the radio system regarding inclusion, limit and quality. Re-enactment of GSM versatile systems is arranged utilizing ATOLL arranging instrument [6]. The remote part in a versatile correspondence arrange costs extraordinary and is of crucial significance to organize quality, so we should make a decent arranging at prior stage, which is useful for organize extension and administration update later on.

The call arrangement achievement rate is one of the most significant key exhibitions. RF improvement is done in this procedure .this procedure includes drive testing, post handling, information examination, suggestion and activity steps. By utilizing appropriate nature of administration the inclusion territory is exceptionally improved. Because of the versatility of endorsers and intricacy of the radio wave proliferation, a large portion of the system issues are brought about by expanding supporters and the evolving condition. RF Optimization is a ceaseless 
procedure that is required as the system advances. RF advancement is completed so as to improve the system execution with the current assets [7].

The Network review is important to pass judgment on the system execution and keep up QOS guidelines. The system review distinguishes irregularities or restrictions in current in general system configuration, assists with improving procedures coming about in enhanced system and improved nature of administration. Here GSM Radio Access organize assessed, and various issues, discoveries, preliminaries and upgrades have been summed up and perceptions/proposals have been inclined to relate the useful parts of RF advancement, which influence the presentation, and QoS of an operational cell arrange. Off-base or missing neighbour relations may make an on-going association drop or be given over to an inappropriate neighbour cell. Extreme neighbour relations in a cell, then again, may cause wrong handover choice with respect to the BSS on account of mistaken estimations. Neighbour relations and boundaries are cross checked to distinguish and evacuate in serious benchmarking, inclusion and quality examination of system is performed with contenders [8].

A recurrence utilized in one cell can be reused in another cell at a specific separation. This separation is called reuse separation. The benefit of advanced framework is that they can reuse frequencies more effectively than the simple ones, for example the reuse separation can be shorter, and the limit expanded. A cell framework is situated in reuse of frequencies. All the accessible frequencies are partitioned into various recurrence gatherings with the goal that a specific recurrence consistently has a place with a specific recurrence gathering. The recurrence bunches together structure a group. "A bunch is a region where all recurrence bunches are utilized once, yet not reused." The frequencies can be separated into various recurrence gatherings. This presents the terms reuse designs and reuse lattices. The most well-known reuse designs in GSM are "4/12" and "3/9". 4/12 implies that the accessible frequencies are isolated into 12 recurrence gatherings, which thus are situated at 4 base stations locales. This accepts the base station has three cells associated with it. The recurrence bunches are frequently allotted a number or name, for example, A1, B1, C1, D1, A2,..... D3. 3/9 implies that the accessible frequencies are separated into 9 recurrence bunches situated at 3 locales. Issue with C/A might show up in specific pieces of a phone, emerging from adjoining frequencies in neighbouring cells [9].

RF Planning is the way toward doling out frequencies, transmitter areas and boundaries of a remote correspondences framework to give adequate inclusion and ability to the administrations required. The RF plan of a cell correspondence framework has two destinations: inclusion and limit. Inclusion identifies with the topographical impression inside the framework that has adequate RF signal solidarity to accommodate a call/information meeting. Limit identifies with the capacity of the framework to support a given number of endorsers. Limit and inclusion are interrelated [10]. To improve inclusion, limit must be yielded, while to improve limit, inclusion should be relinquished.

\section{Proposed Methodology}

The Solution to overcome the interference and hand over problems is to automate the works of mobile switching centre (MSC) like analyse call destinations, route calls, handling signalling, control handover, compress and crypt voice. Using Atoll with the features like automatic frequency planning (AFP) and automatic cell planning (ACP ), proper coding is fed into the Software development kit (SDK) and that will make the mobile switching centre (MSC) works automatically. Through this approach, interference and hand over problems can be easily managed by using software development kit (SDK) in Atoll planning tool. Loss of data, traffics, interference is not possible by the automation in the Software development kit (SDK) module. Handover problems can be overcome through the proper frequency reuse of site-location by using orthogonal frequency division multiplexing (OFDM) technique. The proposed work flow is shown in figure 2. The proposed system's features are:

a) Interference and hand over problems can be overcome.

b) Network will work efficiently. 
c) Automatically monitors the works of mobile switching centre (MSC) and no manual calculations are needed.

d) Easy to handle problems and certain steps can be easily taken.

e) Easy maintenance.

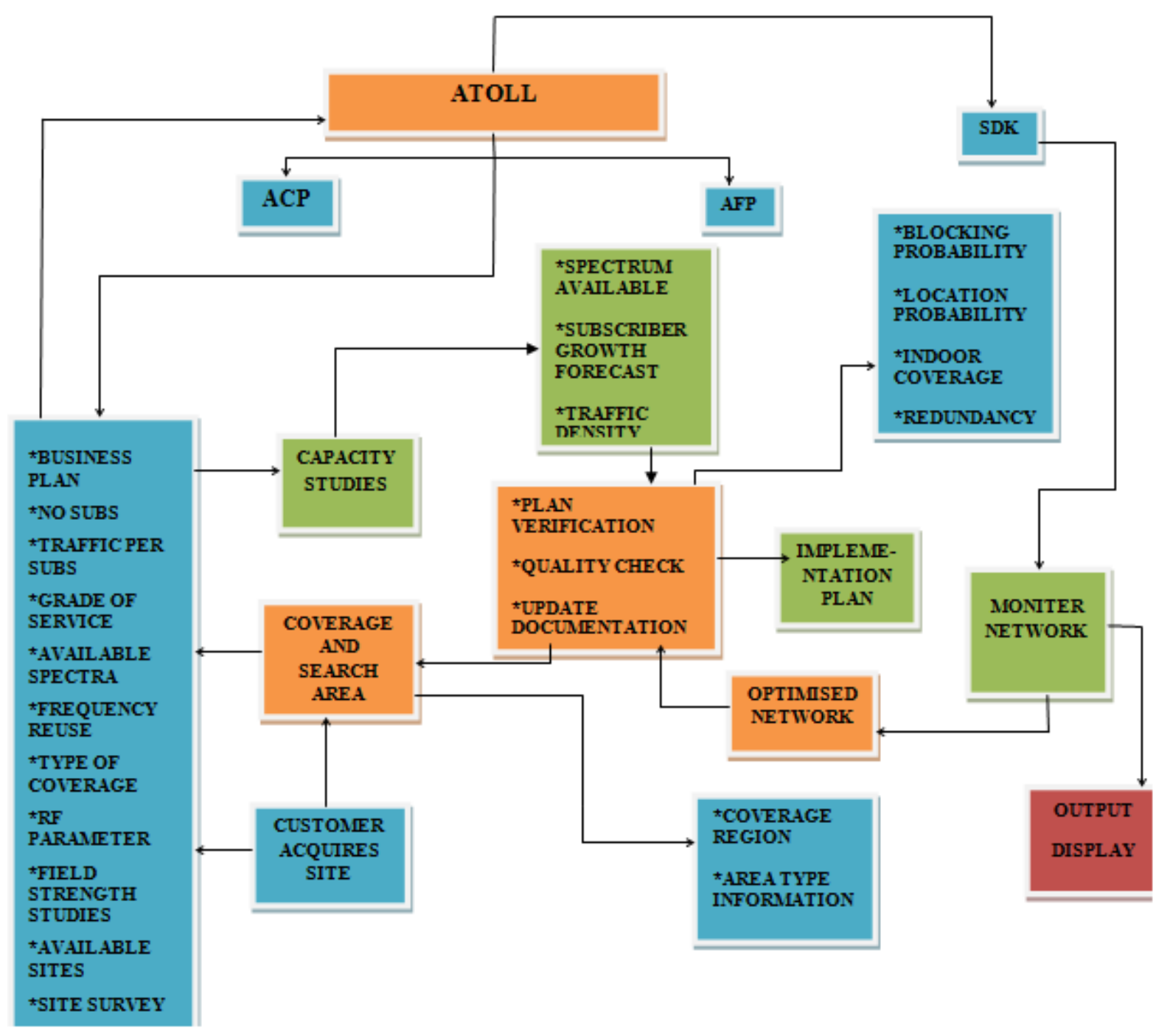

Fig 2: Structure of proposed RF plan of LTE network using ATOLL

The principal level of the RF arranging process is a budgetary level. It utilizes the RF Link Budget alongside a measurable proliferation model (for example Hata, COST-231 Hata or Erceg-Greenstein) to surmised the inclusion region of the arranged locales and to in the end decide what number of destinations are required for the specific RF correspondence framework. The factual proliferation model does exclude landscape impacts and has an incline and block an incentive for each kind of condition (Rural, Urban, Suburban, and so forth.). The second degree of the RF Planning process is an increasingly nitty gritty proliferation model. Programmed arranging instruments are regularly utilized in this stage to perform definite forecasts.

The engendering model considers the qualities of the chose radio wire, the territory, and the land use and land mess encompassing each site. Since these elements are thought of, this spread model gives a superior gauge of the inclusion of the destinations than the underlying measurable engendering model. In this manner, its utilization, related to the RF interface financial plan, creates an increasingly precise assurance of the quantity of locales required. The third period of the RF arranging process consolidates further detail into the RF plan. This 
stage incorporates things, for example, gathering drive information to be utilized to tune or align the spread forecast model, anticipating the accessible information throughput at each site, tweaking of boundary settings (for example receiving wire direction, down inclining, recurrence plan). This procedure is required in the organization of the framework or in deciding assistance contract based inclusion.

\section{Simulation Results}

Atoll is RF arranging and enhancement programming. In 1997, Forsk discharged the primary adaptation of Atoll, its leader RF arranging programming. From that point forward, Atoll has remained the most exceptional $\mathrm{RF}$ arranging and improvement stage accessible and has arrived at a main situation in the market with more than 3500 introduced licenses. Atoll Core is the focal module that bolsters the UI, the GIS includes, the proliferation displaying motor, all information the executives administrations, interfaces and programming advancement devices. The last period of the RF arranging process includes persistent enhancement of the RF intend to oblige for changes in the earth or extra help necessities (for example extra inclusion or limit). This stage begins from starting system arrangement and includes gathering estimation information all the time that could be by means of drive testing or incorporated assortment. The information is then used to design new destinations or to improve the boundary settings (for example reception apparatus direction, down inclining, recurrence plan) of existing destinations. The reference obstruction proportion is characterized in GSM as the impedance proportion for which the necessary execution as far as casing deletion, bit mistake rate or remaining piece blunder rate is met.

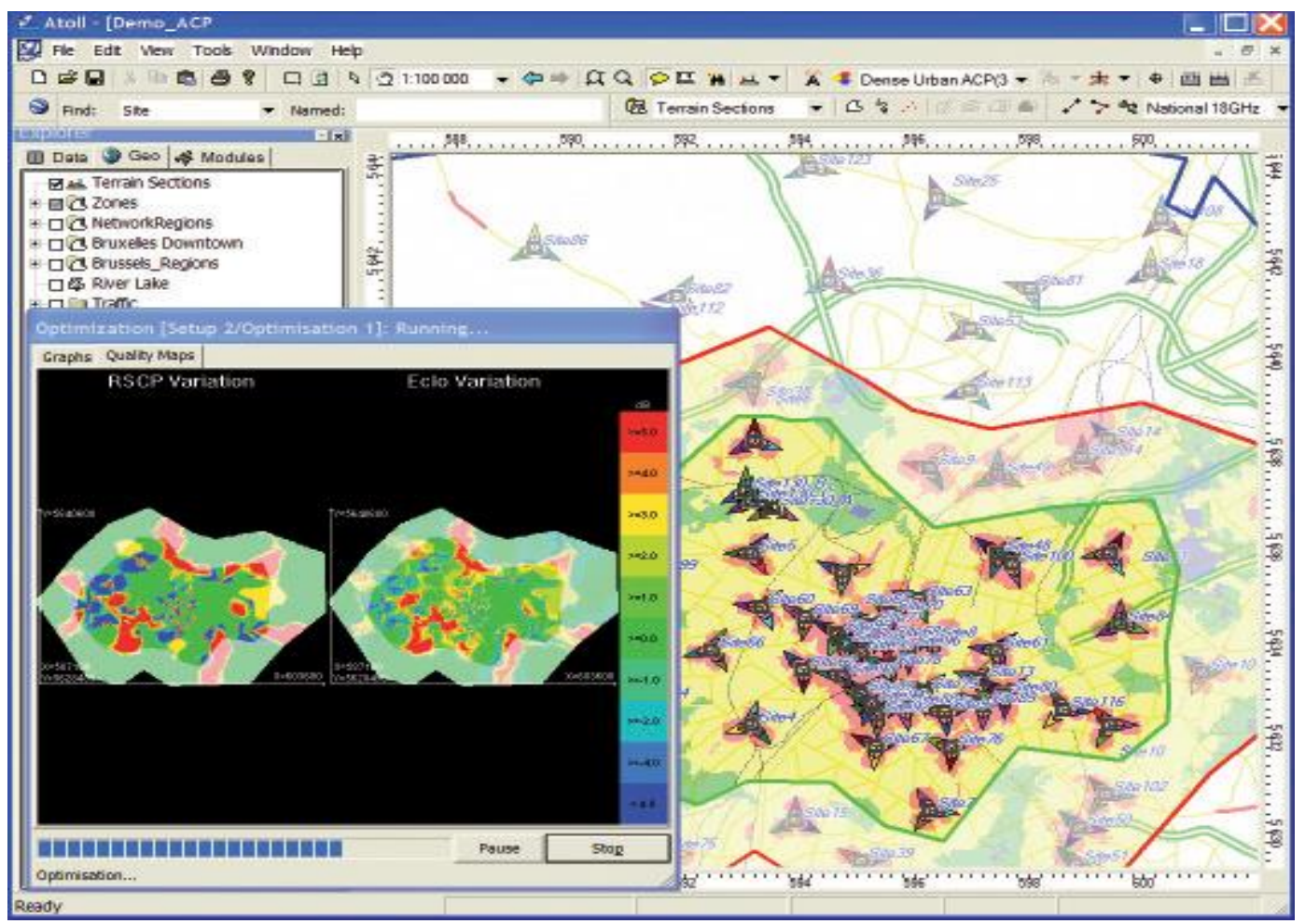

Fig 3: Simulation result showing proposed RF plan for the LTE network

From the inclusion zones determined by the assistance of computerized maps it is very simple to acquire the normal obstruction territories. Since the recurrence plan is still to be done, the numerous obstructions can't be determined. Subsequently the procedure works for each pair of BS checking the proportion between the twosignal pixels. The likelihood of future different obstruction can be decreased by including some edge, say $6 \mathrm{~dB}$ to the reference impedance proportion. On the off chance that the percent of the meddled territory is bigger than a given predefined level, the pair can't work in a similar channel. The choices about handover are commonly taken care of by the RNC. It constantly screens data with respect to the signs being gotten by both the UE and 
Node B and when a specific connection has fallen under a given level and another better radio channel is accessible, it starts a handover. As a feature of this observing procedure, the UE gauges the Received Signal Code Power (RSCP) and Received Signal Strength Indicator (RSSI) and the data is then come back to the hub B and henceforth to the RNC on the uplink control channel.

Connection spending computations are accomplished for both the uplink and downlink. As the force transmitted by the portable station reception apparatus is not exactly the force transmitted by the base station radio wire, the uplink power spending plan is more basic than the downlink power financial plan. Therefore, the affectability of the base station in the uplink bearing gets one of the basic factors all things considered identified with gathering of the force transmitted by the versatile station reception apparatus. In the downlink course, transmitted force and the additions of the receiving wires are significant boundaries. As far as misfortunes in the gear, the consolidated misfortune and the link misfortune are to be thought of. Combiner misfortune comes just in the downlink estimations while the link misfortune must be joined in the two headings. For the other gear (for example the MS), the transmitted force in the uplink course is significant. To get the sign transmitted from the BTS reception apparatus even in far off regions, the affectability of the MS becomes possibly the most important factor. The transmitting and the getting receiving wire gains and the link misfortune boundaries are to be considered on the BTS.

Table 1: Table showing simulation results

\begin{tabular}{|l|c|c|c|}
\hline Parameters & Urban High Density & $\begin{array}{l}\text { Urban and Sub Urban } \\
\text { High density }\end{array}$ & $\begin{array}{l}\text { Sub Urban and rural } \\
\text { density }\end{array}$ \\
\hline Total TCH & 21 & 14 & 7 \\
\hline TFC/BTS & 42.12 & 24.6 & 8.82 \\
\hline BCCH & 24 & 28 & 35 \\
\hline No. of BTS & 14.245 & 60.97 & 79.36 \\
\hline Non-BCCH & 21 & 24 & 26 \\
\hline
\end{tabular}

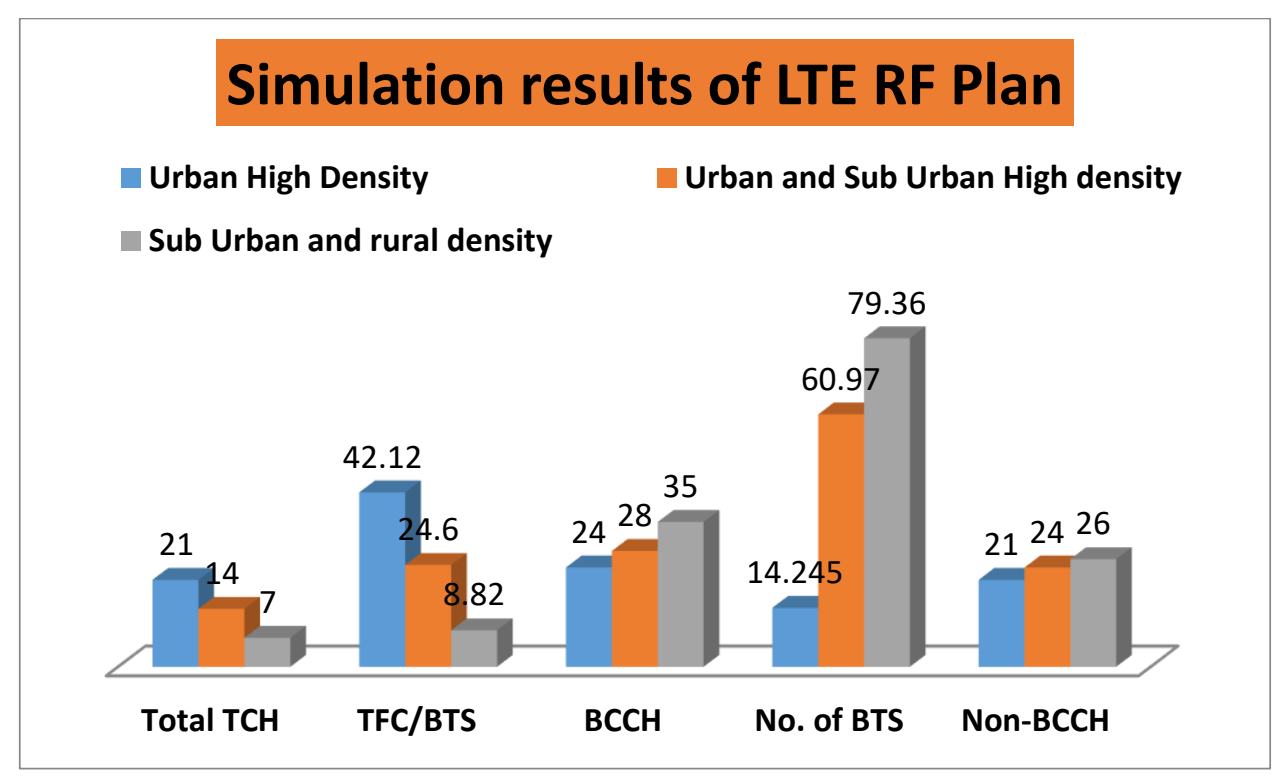

Fig 4: Chart showing simulation parameters of proposed RF plan for the LTE network

The accessibility of these limit redesign arrangements relies upon the base station maker. All these limit update choices may not be accessible in all base station types. These limit overhaul arrangements don't require any progressions to the reception apparatus designs, just redesigns inside the base station bureau are required on the site. The uplink inclusion isn't influenced by these updates. The system execution can be best seen when the system load is high. With low burden a portion of the issues may not be obvious. In this manner, we have to 
consider fake burden age to imitate high stacking in the system. A high uplink burden can be produced by expanding the Eb/N0 focus of the external circle power control. In the typical activity the external circle power control furnishes the necessary quality with least $\mathrm{Eb} / \mathrm{NO}$. In the event that we increment physically the Eb/N0 target, for example $10 \mathrm{~dB}$ higher than the ordinary activity point, that uplink association will cause multiple times more impedance and changes over $32 \mathrm{kbps}$ association into $320 \mathrm{kbps}$ high piece rate association from the obstruction perspective.

\section{Conclusion}

The RF planning for LTE network with hybrid parameters were successfully proposed in the research article. Finally 93 BTS were taken into consideration of population density as 600 Erlangs for Urban high density, 1500 Erlangs for Urban and sub urban, 700 Erlang for rural density in respect of Coimbatore SSA. The radio frequency planning GSM network was thus performed for the best signal reception and C/I ratio to provide optimum coverage and high reliability at low cost. No presumptions are made in the size, shape, arrangement or the situation of cell size. Utilization of direct drive-test information that mirrors all the non-consistency and capriciousness of the genuine RF condition. The new calculation procedures can deal with the multifaceted nature of the real territory. The re-use of the site frequency \& network structure will enhance the quality of service. The subscriber addition, traffic management will be very easy to implement. The increased picture quality and speech quality in the network will be possible.

\section{References}

[1] Alexei barbosa de aguiar, (2009), 'A Novel model for optimized GSM network design', IJCSIS, Volume 4, issue 2, pp. 17-23.

[2] Jahangir khan, (2010), 'Handover management in GSM cellular system', IJCA, Volume 8, issue no. 12, pp. 1-6.

[3] Reshma begum shaik, (July 2012), 'Simulation of GSM mobile networks planning using ATOLL planning tool', IJEIT, Volume 2, issue 1, pp. 1340-1350.

[4] Venkatasai Sireesha.B, (2013), 'Increasing of call success rate in GSM service area using RF optimization', IJERA, Volume 1, issue 4, pp. 1479-1485.

[5] M. Golio, "Strategic Planning for RF Technologies - Implications for 5G and IoT Emerging Radio Products," 2018 IEEE MTT-S Latin America Microwave Conference (LAMC 2018), Arequipa, Peru, 2018, pp. 1-4, doi: 10.1109/LAMC.2018.8699035.

[6] P. Kar and B. Dappuri, "Site Survey and Radio Frequency Planning for the Deployment of Next Generation WLAN," 2018 International Conference on Wireless Communications, Signal Processing and Networking (WiSPNET), Chennai, 2018, pp. 1-4, doi: 10.1109/WiSPNET.2018.8538731.

[7] B. Yee et al., "RF Propagation Measurements Using a Mobile Receiver System," 2018 IEEE International Symposium on Antennas and Propagation \& USNC/URSI National Radio Science Meeting, Boston, MA, 2018, pp. 87-88, doi: 10.1109/APUSNCURSINRSM.2018.8608255.

[8] A. H. A. El-Malek, A. M. Salhab, S. A. Zummo and M. Alouini, "Effect of RF Interference on the Security-Reliability Tradeoff Analysis of Multiuser Mixed RF/FSO Relay Networks With Power Allocation," in Journal of Lightwave Technology, vol. 35, no. 9, pp. 1490-1505, 1 May1, 2017, doi: 10.1109/JLT.2017.2687863.

[9] M. R. Tanhatalab, S. M. J. J. Jadeh and A. O. Esfahani, "Practical achievements on throughput improvement in real networks," 2017 International Symposium on Wireless Systems and Networks (ISWSN), Lahore, 2017, pp. 1-6, doi: 10.1109/ISWSN.2017.8250037.

[10] S. S. Sarnin, M. Y. Yusof, M. Y. Mohamad, W. N. W. Mohamad, N. F. Naim and N. Ya'acob, "Multibeam RF Antenna Solution for Capacity Enhancement in Stadium Shah Alam," 2018 9th IEEE Control and System Graduate Research Colloquium (ICSGRC), Shah Alam, Malaysia, 2018, pp. 197200, doi: 10.1109/ICSGRC.2018.8657494. 